\title{
USING MARYLIE WITH THE PARTICLE BEAM OPTICS LABORATORY
}

\author{
George H. Gillespie, Barrey W. Hill, Hendy Martono, John M. Moore \\ G. H. Gillespie Associates, Inc., P.O. Box 2961, Del Mar, CA 92014, U.S.A. \\ Alex J. Dragt \\ Department of Physics, University of Maryland, College Park, MD 20742, U.S.A.
}

\begin{abstract}
A MARYLIE module has been developed for a new version of the Particle Beam Optics Laboratory (PBO $\mathrm{Lab}^{\mathrm{TM}}$ ). MARYLIE is an optics code based on a Lie algebra formulation of charged particle trajectory calculations and is particularly useful for particle tracking and for the analysis of linear and nonlinear lattice properties. The PBO Lab provides an intelligent graphic user interface based upon the Multi-Platform Shell for Particle Accelerator Related Codes (S.P.A.R.C. MP), a software framework developed specifically to support accelerator modeling, simulation and training. Transport element icons are selected from a palette and assembled into beamlines by graphical construction. Optical cells and lattices composed of element groups may be defined as sublines, and elements or sublines can be replicated using an alias element. An icon-based description of MARYLIE commands and procedural processes has also been developed. The icon-based beamlines and commands generate entries for the MARYLIE Master Input File (MIF). Frequent computations are encapsulated into interactive commands which create the needed entries in the MIF, call MARYLIE to execute the required computations, and then return output data to the graphic interface for display. Use of the PBO Lab MARYLIE module is described and illustrations from the Windows95 implementation are presented.
\end{abstract}

\section{INTRODUCTION}

The MARYLIE program [1] is a powerful tool for studying nonlinear optics. The version of MARYLIE 3.0 described in [2] is being integrated with PBO Lab 2.0 [3] to provide a unique graphical environment for beamline studies. PBO Lab provides an easy-to-use graphic user environment, customized to the needs of the accelerator community for both particle optics education [4] and beamline design [5]. This paper discusses selected features of the new MARYLIE Module.

\section{MARYLIE MODULE FOR PBO LAB}

The use of the PBO Lab MARYLIE Module is perhaps best illustrated via an example: designing and then studying the performance of a four-cell, second-order achromatic bend. (It should be noted that MARYLIE can also be used to design a third-order achromat.) The basic layout of the example beamline is illustrated in Figure 1, using the iconic representation provided by the PBO Lab graphical beamline construction kit. The beamline is composed of four identical cells, with each cell containing a sector bend, two quadrupoles and two sextupoles, all interspersed with drifts. Figure 1 also illustrates two approaches (described below) for using the MARYLIE Module to fit magnet strengths.

(a) Achromat cell layout

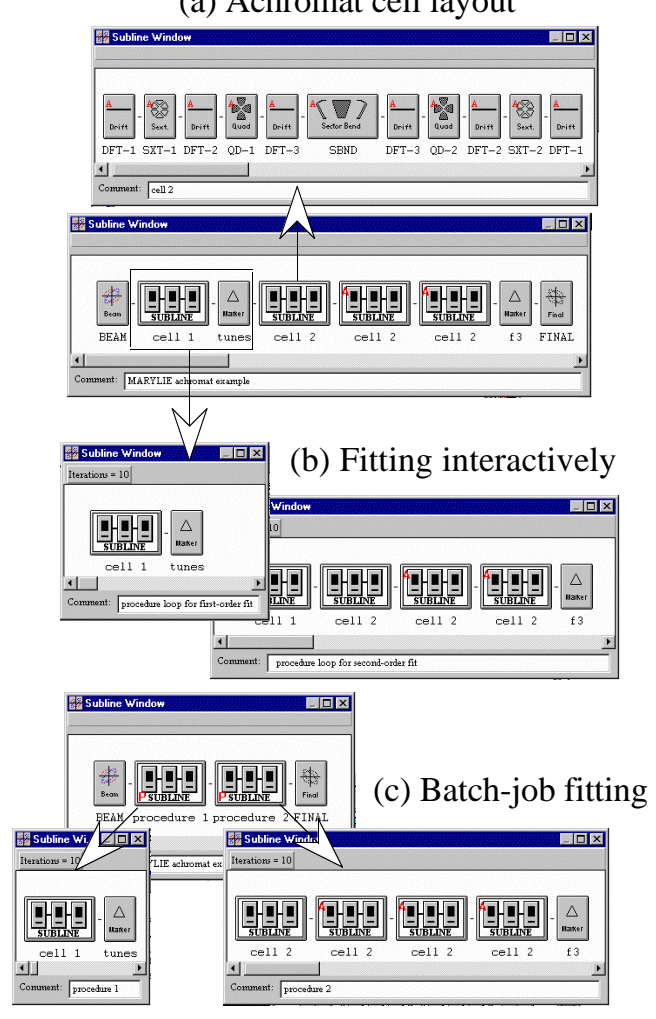

Figure 1. Selected images from the PBO Lab MARYLIE Module showing icon representations of: (a) the four-cell achromat, (b) creation of MARYLIE procedure loops used to fit first-order and second-order optical constraints interactively, and (c) the use of two MARYLIE procedure loops to fit both sets of conditions as a "batch job." All images are from the Windows95/98/NT implementation.

The construction of a scalable version of this type of achromatic bend had been discussed previously $[5,6]$. With the PBO Lab, formulas can be used to incorporate dependent parametric relationships between elements [5], while intelligent alias elements replicate either identical or near-identical elements with no redundant data $[3,6]$. The graphical construction of a beamline defines all data necessary to run any of the particle optics modules 
installed in the PBO Lab. The PBO Lab takes care of all $\mathrm{I} / \mathrm{O}$ requirements for each code, with no knowledge required of the user concerning command syntax, file formats, or similar code-specific details.

The principal design tasks are to determine: (a) the quadrupole strengths that are necessary for each cell to have phase advances of $90^{\circ}$ in both transverse directions (i.e. quarter-wave transformer), and (b) the sextupole strengths that are required to eliminate (second-order) chromatic aberrations. The primary performance studies involve simulating beams with different momentum spreads passing through the beamline. MARYLIE can perform both of these functions (as well as many more). The remainder of this paper illustrates how this is done using the PBO Lab.

\subsection{Solving Fitting Problems}

Fitting with MARYLIE is done using procedure loops [1]. A procedure loop is readily defined graphically in the PBO Lab: the user selects (with the mouse) a beamline segment containing one or more Marker Pieces, and then chooses the "Create Procedure Loop" option from the Commands menu. The steps are similar to the actions used to interactively create a Map Piece [6]. Figure 1(b) illustrates the selection of elements and creation of the procedure loop for fitting the first-order properties of the achromat. Desired optical conditions are set using Marker Pieces in a procedure loop, while the parameters to be varied are set using "S" buttons in the Piece Windows of elements, similar to those of the PBO Lab TRANSPORT Module [5]. The first Marker Piece is used to specify the desired phase advances for the first cell, and the field gradients of the first two Quad Pieces are selected as variation parameters. MARYLIE uses tunes $v$, related to the phase advances $\mu$ by $\nu=\mu /(2 \pi)$, so for transverse phase advances of $\pi / 2$ the MARYLIE fitting aims are:

$$
v_{\mathrm{x}}=0.25 \text { and } v_{\mathrm{y}}=0.25
$$

The first-order fit may be carried out interactively, and then the Lie Map coefficients examined to select the largest non-linear terms to minimize as a second step. This is easily done using a graphic display of the polynomial coefficients for a Map Piece of the beamline. The desired fitting conditions for second-order are:

$$
\mathrm{f}_{3}(33)=0 \quad \text { and } \quad \mathrm{f}_{3}(67)=0
$$

The first procedure loop is removed and a second procedure loop containing all four cells is then defined. This loop is also shown in Figure 1(b), with the aims (2) specified using the Marker Piece at the end of the achromat, and the first two sextupole strengths selected as fitting variables. Alternatively, two separate procedure loops may be created as illustrated in Figure 1(c), to define a single "batch job," which will sequentially carry out both the first- and second-order fitting using a single interactive command.

\subsection{Comparison of Results to Other Calculations}

The results from MARYLIE may be readily compared to results from any other code integrated with PBO Lab 2.0. The ease of carrying out such comparisons is difficult to over-emphasize. For example, to compare beamline transfer maps or output beam distributions, one simply selects a different sub-menu from the Commands Menu [3] and executes an appropriate command. The PBO Lab takes care of correctly formatting the input to each code, executing the selected command, and displaying the results. Appropriate TRANSPORT fitting constraints equivalent to those given by (1) and (2) are easily defined with PBO Lab [5]:

$$
\begin{array}{rll}
\mathrm{R}_{11}+\mathrm{R}_{22}=0 & \text { and } & \mathrm{R}_{33}+\mathrm{R}_{44}=0, \\
\mathrm{~T}_{126}=0 & \text { and } & \mathrm{T}_{346}=0 .
\end{array}
$$

Table 1 summarizes the fitting results obtained for the quadrupole and sextupole field strengths using the MARYLIE and TRANSPORT Modules of PBO Lab 2.0.

Table 1. Comparison of quadrupole and sextupole fitting results using the MARYLIE and TRANSPORT Modules with PBO Lab 2.0 for the achromatic bend described in reference [5]. Tolerances for all aims were set at $10^{-6}$.

\begin{tabular}{ccccccccc}
\hline Computation Engine & \multicolumn{2}{c}{ Quadrupole 1 (B') } & \multicolumn{2}{c}{ Quadrupole 2 (B') } & Sextupole 1 (S) & \multicolumn{2}{c}{ Sextupole 2 (S) } \\
\hline MARYLIE & 9.6843595 & $\mathrm{~T} / \mathrm{m}$ & -10.180100 & $\mathrm{~T} / \mathrm{m}$ & 107.41 & $\mathrm{~T} / \mathrm{m}^{2}$ & -146.74 & $\mathrm{~T} / \mathrm{m}^{2}$ \\
TRANSPORT & 9.6843590 & $\mathrm{~T} / \mathrm{m}$ & -10.180092 & $\mathrm{~T} / \mathrm{m}$ & 107.04 & $\mathrm{~T} / \mathrm{m}^{2}$ & -145.67 & $\mathrm{~T} / \mathrm{m}^{2}$ \\
\hline
\end{tabular}

\subsection{Performance Simulations of Beamlines}

Once the fitting tasks are complete, MARYLIE may then be used to simulate the performance of the achromat for different initial beam conditions. Several ray tracing and tracking options are available in MARYLIE. Figure 2 illustrates results using the element-by-element ray tracing command. Six output beam cross sections are shown: three for the achromat with the sextupole correctors turnedoff, and three with the sextupole strengths set to the values given in Table 1. The initial beam for all cases was a 6-D uniformly filled ellipsoid of 1000 particles with semi-axes parameters from Table 1 of reference [7]. Results are shown for three different initial momentum spreads. The second-order achromat performs very well for this beam with a $9 \%$ momentum spread, nearly identical to that for a beam with $3 \%$ momentum spread, and may be adequate for use with momentum spreads up to $27 \%$, depending upon the application. 


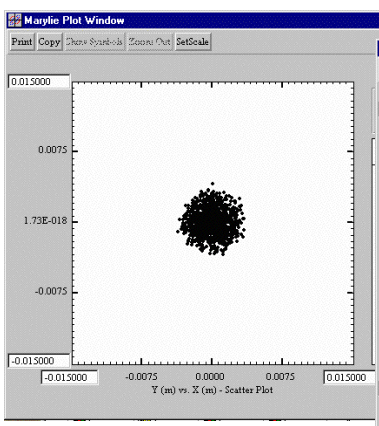

(a)

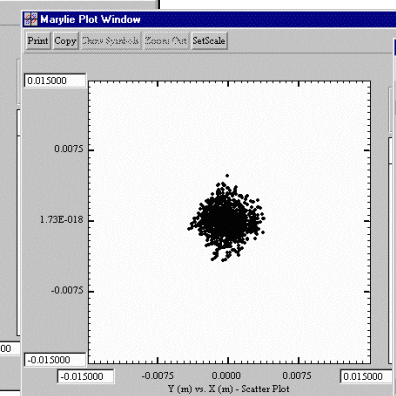

(b)
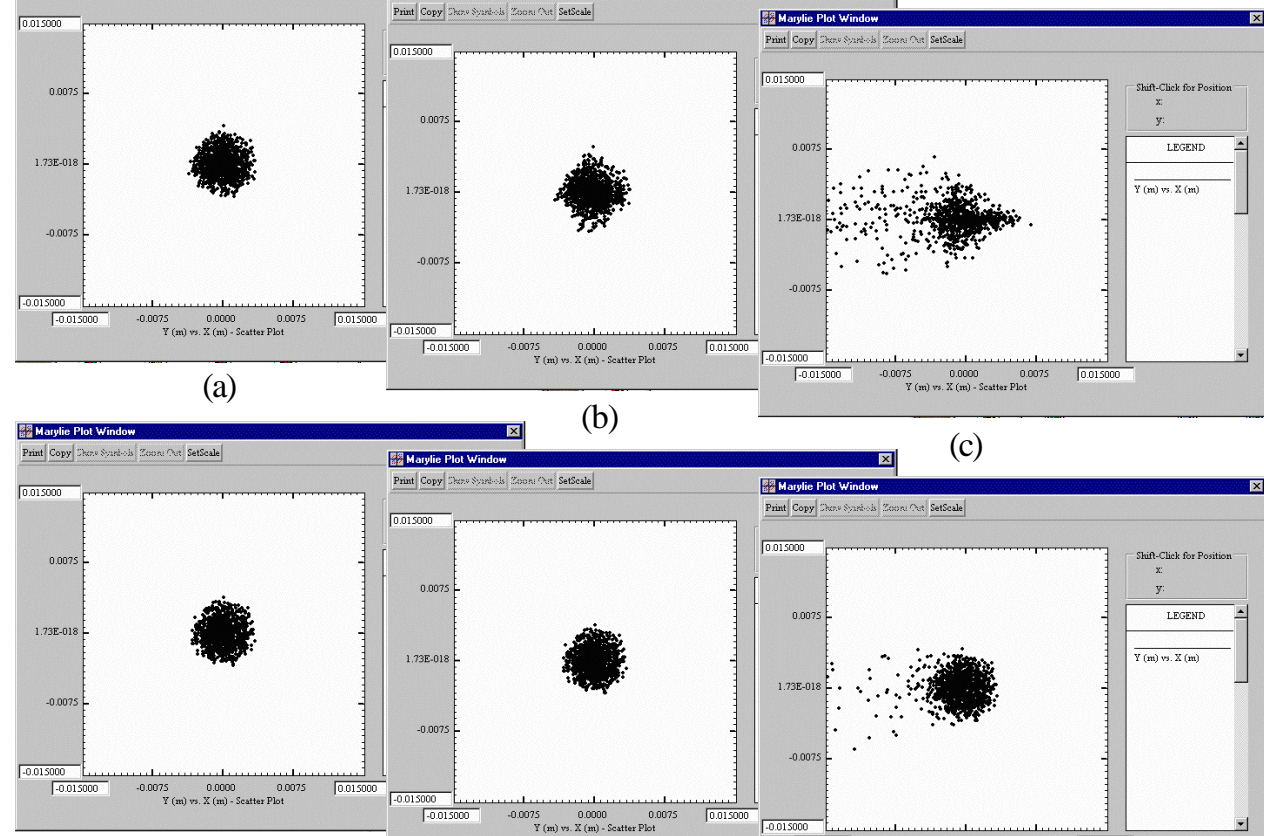

(d)

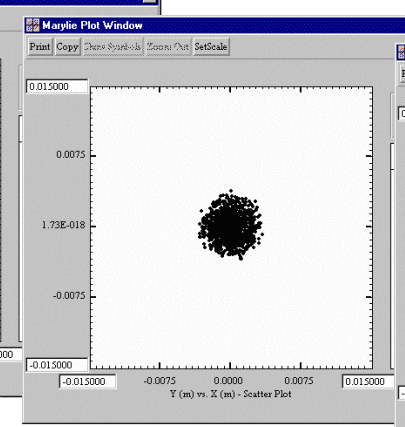

(e) (c)

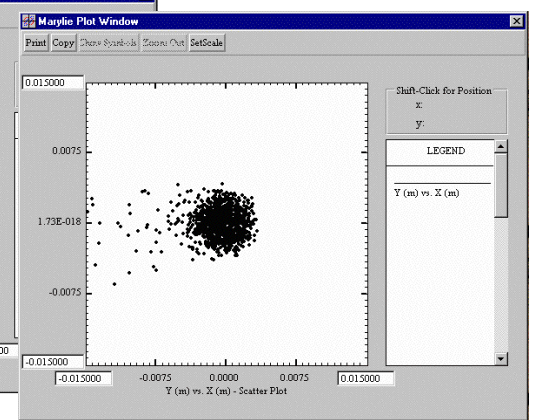

(f)

Figure 2. Two-dimensional scatter plots of the beam cross-section at the end of the achromatic bend, without sextupole correctors [top (a)-(c)] and with sextupole correctors [bottom (d)-(f)]. In both cases, results are shown for three different values of the initial momentum spread $\delta: 3 \%, 9 \%$ and $27 \%$, increasing from left to right. Window images are from the PBO Lab 2.0 MARYLIE Module as implemented for Windows95/98/NT platforms.

\section{SUMMARY}

An innovative MARYLIE Module for the PBO Lab [8] has been developed that provides an easy-to-use graphic interface for setting up problems and executing commands. The unique S.P.A.R.C. MP framework [3] provides both for the graphical construction of beamlines and for graphically formulating MARYLIE procedures.

\section{ACKNOWLEDGEMENTS}

Work supported in part by the U. S. Department of Energy under Small Business Innovation Research (SBIR) Grant No. DE-FG03-95ER81975.

\section{REFERENCES}

[1]A. J. Dragt, D. R. Douglas, F. Neri, C. T. Mottershead, R. D Ryne, E. Forest, L. M Healy, P. Schutt and J. van Zeijts, "MARYLIE 3.0 User's Manual, A Program for Charged Particle Beam Transport Based on Lie Algebraic Methods," February draft, 721 pages (1999).

[2] A. J. Dragt, "Release of MARYLIE 3.0," this conference (1999).

[3] B. W. Hill, H. Martono, J. M. Moore and J. S. Gillespie, "An Application Framework and Intelligent Graphic User Interface for Multiple Accelerator Codes," to be published in the proceedings of the
International Computational Accelerator Physics Conference (Monterey), 4 pages (1998).

[4] N. A. Brown, G. H. Gillespie, B. W. Hill, M. C. Lampel, H. Martono and J. M. Moore, "The Particle Beam Optics Laboratory (PBO LAB ${ }^{\mathrm{TM}}$ ): A New Education and Training Aid," Proc. 1998 European Particle Accelerator Conference, 1183-1185 (1998).

[5] G. H. Gillespie, B. W. Hill, H. Martono, J. M. Moore, M. C. Lampel, and N. A. Brown, "Using the Particle Beam Optics Laboratory (PBO $\mathrm{LAB}^{\mathrm{TM}}$ ) for Beamline Design and Simulation," to be published in the proceedings of the 15th International Conference on Cyclotrons and Their Applications, 4 pages (1998).

[6] G. H. Gillespie, B. W. Hill, M. C. Lampel, H. Martono, J. M. Moore, A. J. Dragt, "Multi-Platform Graphic User Interface for MARYLIE Charged Particle Beam Transport Code," to be published in the proceedings of the International Computational Accelerator Physics Conf. (Monterey), 5 pages (1998).

[7] G. H. Gillespie, B. W. Hill and M. C. Lampel, "Using 3-D Perspectives as a Visualization Tool for Phase Space Data," to be published in the proceedings of the International Computational Accelerator Physics Conference (Monterey), 5 pages (1998).

[8] The $P B O L a b^{\mathrm{TM}}$ is available from AccelSoft Inc., www.ghga.com/accelsoft. 